\title{
Asymptotic Smoothing and Global Attractors for a Class of Nonlinear Evolution Equations
}

\author{
Yongqin Xie, ${ }^{1}$ Zhufang $\mathrm{He}^{2}{ }^{2}$ Chen $\mathrm{Xi}^{2}{ }^{2}$ and Zheng Jun ${ }^{2}$ \\ ${ }^{1}$ School of Mathematics and Computing Science, Changsha University of Science and Technology, Changsha 410004, China \\ ${ }^{2}$ Hunan Provincial Center for Disease Control and Prevention, Changsha, Hunan 410005, China \\ Correspondence should be addressed to Yongqin Xie; yqxie_11@163.com
}

Received 31 March 2013; Accepted 24 April 2013

Academic Editors: S. Liu and W. Shen

Copyright (c) 2013 Yongqin Xie et al. This is an open access article distributed under the Creative Commons Attribution License, which permits unrestricted use, distribution, and reproduction in any medium, provided the original work is properly cited.

\begin{abstract}
We prove the asymptotic regularity of global solutions for a class of semilinear evolution equations in $H_{0}^{1}(\Omega) \times H_{0}^{1}(\Omega)$. Moreover, we study the long-time behavior of the solutions. It is proved that, under the natural assumptions, these equations possess the compact attractor $\mathscr{A}$ which is bounded in $H^{2}(\Omega) \times H^{2}(\Omega)$, where the nonlinear term $f$ satisfies a critical exponential growth condition.
\end{abstract}

\section{Introduction}

In this paper, we study the asymptotic regularity and the longtime behaviors of the solutions for the following semilinear evolution equations:

$$
\begin{gathered}
\left(\left|u_{t}\right|^{r-2} u_{t}\right)_{t}-\Delta u-\mu \Delta u_{t}-\Delta u_{t t}+f(u)=g, \quad \text { in } \Omega, \\
\left.u\right|_{t=0}=u_{0},\left.\quad u_{t}\right|_{t=0}=u_{1} \quad \text { in } \Omega, \\
u=0 \quad \text { on } \partial \Omega,
\end{gathered}
$$

where $\Omega$ is an open-bounded set of $\mathbb{R}^{3}$ with smooth boundary $\partial \Omega, \mu>0$, and $r$ satisfies

$$
3 \leq r \leq 6
$$

Equation (1), which appears as a class of nonlinear evolution equations as $r=2$, is used to represent the propagation problems of lengthways wave in nonlinear elastic rods and ion-sonic of space transformation by weak nonlinear effect (see, for instance, [1-4]):

$$
u_{t t}-\Delta u-\mu \Delta u_{t}-\Delta u_{t t}+f(u)=g,
$$

and as $r=3$ (named the Karman equation) is used to represent the flow of condensability airs in the across velocity of sound district (see, for instance, [5])

$$
u_{t} u_{t t}-u_{x x}=0 \text {. }
$$

In [6], the authors have discussed the existence of global solutions in $H_{0}^{1}(\Omega) \times H_{0}^{1}(\Omega)$ under the assumptions that the initial values are sufficiently small. In $[7,8]$, the authors have discussed the nonexistence of global week solutions for the following system:

$$
P\left(u_{t}\right)_{t}+A u+Q\left(t, u_{t}\right)=f(u)
$$

where $P, A$, and $Q$ are nonlinear operators. As $r=2$, in [9], the authors have discussed the long-time behaviors of solutions of (1) in $H_{0}^{1}(\Omega) \times H_{0}^{1}(\Omega)$; specifically in [10], the authors have discussed the long-time behaviors of solutions of (1) in $W_{0}^{1, p}(\Omega) \times W_{0}^{1, p}(\Omega)$. However, an open question remains whether the global attractor regularizes in the critical case and as $3 \leq r \leq 6$, the long-time behaviors of solutions of (1) have not been considered completely up to now. In this paper, we try to discuss the problem.

In the study of the global attractor regularization, the critical nonlinearity exponent brings a difficulty. About the regularity of attractor for the strongly damped wave equations, for the subcritical case, the authors in [11] have proved that the global attractor is bounded in $H^{2} \times H^{1}$. For the critical case, Pata and Zelik [12] have proved that the global attractor is bounded in $H^{2} \times H^{2}$ when the nonlinearity $f(\cdot)$ satisfies $\lim \inf _{|s| \rightarrow \infty}\left(f^{\prime}(s) / s\right)>-\lambda_{1}$, for all $s \in \mathbb{R}$, and the authors also pointed out further that one can prove the regularity of the attractor when $f(\cdot)$ only satisfies the natural assumptions 
(which have been realized recently in $[13,14]$ ). A general way is to obtain higher regularity of the solutions than their initial values (see, for instance, $[11,12]$ ). Then we can get the global attractor regularization. However, since (1) contains terms $\left(\left|u_{t}\right|^{r-2} u_{t}\right)_{t}(r>2)$ and $\Delta u_{t t}$, they are essentially different from usual strongly damped wave equations and it is very difficult for us to obtain better regularity of the solutions of (1). Therefore, we must propose a new and more general way to study the smoothing of global attractors for (1).

In this paper, we will apply the techniques introduced in Zelik [15], Sun and Yang [13], and Yang and Sun [14] to overcome the difficulty due to the critical nonlinearity and establish the asymptotic regularity of solutions. Based on this regularity result, we obtain asymptotically compactness of the semigroup $\{S(t)\}_{t \geq 0}$ in $H_{0}^{1}(\Omega) \times H_{0}^{1}(\Omega)$, and the existence of the compact attractor $\mathscr{A}$ has been proved. Moreover the compact attractor $\mathscr{A}$ is bounded in $H^{2}(\Omega) \times H^{2}(\Omega)$.

\section{Functional Setting}

In what follows, we give some notations which will be used throughout this paper. Let $\Omega$ be a bounded subset of $\mathbb{R}^{3}$ with a sufficiently smooth boundary $\partial \Omega, V=H_{0}^{1}(\Omega)$ and $H=L^{2}(\Omega)$ corresponding norms, $\|u\|_{0}=\left(\int_{\Omega}|\nabla u|^{2}\right)^{1 / 2}$, and $|u|_{2}=\left(\int_{\Omega}|u|^{2}\right)^{1 / 2}$, respectively; the norms in $L^{p}(\Omega)(3 \leq p<$ $\infty)$ are denoted by $|u|_{p}=\left(\int_{\Omega}|u|^{p}\right)^{1 / p}$. Let $A$ be the (strictly) positive operator on $L^{2}(\Omega)$ defined by

$$
A=-\Delta \text { with domain } D(A)=H^{2}(\Omega) \cap H_{0}^{1}(\Omega) .
$$

For the family of Hilbert spaces $D\left(A^{s / 2}\right), s \in \mathbb{R}$, their inner products and norms are, respectively,

$$
\langle\cdot, \cdot\rangle_{s}=\left\langle A^{s / 2}, A^{s / 2}\right\rangle, \quad\|\cdot\|_{D\left(A^{s / 2}\right)}=\left|A^{s / 2}\right|_{2} \cdot
$$

Then we have the continuous embedding

$$
\begin{gathered}
D\left(A^{s / 2}\right) \hookrightarrow D\left(A^{r / 2}\right) \quad \text { for any } s>r, \\
D\left(A^{s / 2}\right) \hookrightarrow L^{6 /(3-2 s)}(\Omega) \quad \text { for any } s \in\left[0, \frac{3}{2}\right) .
\end{gathered}
$$

The product Hilbert spaces

$$
E_{s}=D\left(A^{(1+s) / 2}\right) \times D\left(A^{(1+s) / 2}\right),
$$

endowed with the usual inner products and norms

$$
\|(u, v)\|_{E_{s}}^{2}=\left|A^{(1+s) / 2} u\right|_{2}^{2}+\left|A^{(1+s) / 2} v\right|_{2}^{2}, \quad \forall(u, v) \in E_{s} .
$$

Denote by $C$ any positive constant, which may be different from line to line and even in the same line.

Throughout the paper we assume that the function $f \in$ $\mathbb{C}^{1}(\mathbb{R}, \mathbb{R})$ satisfies the following conditions:

$$
\left|f^{\prime}(s)\right| \leq C\left(1+|s|^{4}\right), \quad \forall s \in \mathbb{R} .
$$

Also, let $f$ admit the decomposition $f=f_{0}+f_{1}$, where $f_{0}, f_{1} \in \mathbb{C}^{1}(\mathbb{R})$, satisfying

$$
\begin{aligned}
& \left|f_{0}(s)\right| \leq C\left(1+|s|^{5}\right), \quad \forall s \in \mathbb{R}, \\
& f_{0}(s) s \geq 0, \quad \forall s \in \mathbb{R}, \\
& \left|f_{1}(s)\right| \leq C\left(1+|s|^{p}\right), \quad p<5, \forall s \in \mathbb{R}, \\
& \limsup _{|s| \rightarrow \infty} \frac{f_{1}(s)}{s}>-\lambda_{1} \text {, }
\end{aligned}
$$

where $\lambda_{1}$ is the first eigenvalue of $-\Delta$ in $H_{0}^{1}(\Omega)$ with the Dirichlet boundary condition. Without loss of generality, we can think $p$ is large enough, say, $p \geq 3$. Notice that by (13) and (15), there exists $\lambda<\lambda_{1}$, such that

$$
f(s) s \geq f_{1}(s) s \geq-\lambda s^{2}-C .
$$

We denote by $F$ the function

$$
F(u)=\int_{\Omega} \int_{0}^{u} f(s) d s,
$$

which is easily seen to satisfy the inequalities

$$
\begin{gathered}
F(u) \geq-\frac{1}{2} \lambda|u|_{2}^{2}-C, \\
\int_{\Omega} f(u) u \geq F(u)-\frac{1}{2} \lambda|u|_{2}^{2}-C .
\end{gathered}
$$

We will complete our task exploiting the transitivity property of exponential attraction [16, Theorem 5.1], which we recall below for the readers' convenience.

Lemma 1 (see [16]). Let $\mathscr{K}_{1}, \mathscr{K}_{2}$, and $\mathscr{K}_{3}$ be subsets of $E_{0}$ such that

$$
\begin{aligned}
& \operatorname{dist}_{E_{0}}\left(S(t) \mathscr{K}_{1}, \mathscr{K}_{2}\right) \leq L_{1} e^{-v_{1} t}, \\
& \operatorname{dist}_{E_{0}}\left(S(t) \mathscr{K}_{2}, \mathscr{K}_{3}\right) \leq L_{2} e^{-v_{2} t},
\end{aligned}
$$

for some $v_{1}, v_{2}>0$ and $L_{1}, L_{2} \geq 0$. Assume also that for all $z_{1}, z_{2} \in \bigcup_{t \geq 0} S(t) \mathscr{K}_{j}(j=1,2,3)$ there holds

$$
\left\|S(t) z_{1}-S(t) z_{2}\right\|_{E_{0}} \leq L_{0} e^{v_{0} t}\left\|z_{1}-z_{2}\right\|_{E_{0}}
$$

for some $v_{0} \geq 0$ and some $L_{0} \geq 0$. Then it follows that

$$
\operatorname{dist}_{E_{0}}\left(S(t) \mathscr{K}_{1}, \mathscr{K}_{3}\right) \leq L e^{-v t},
$$

where $v=v_{1} v_{2} /\left(v_{0}+v_{1}+v_{2}\right)$ and $L=L_{0} L_{1}+L_{2}$.

\section{Global Solution in $H_{0}^{1}(\Omega) \times H_{0}^{1}(\Omega)$}

Exploiting the dissipative conditions (16) and (18) and the standard energy estimates technique, it is easy to obtain the following result.

Lemma 2. Assume that

$$
\left\|\left(u_{0}, u_{1}\right)\right\|_{0}<R
$$


for some $R>0$. Then one has the following estimates:

(i) $\left|u_{t}(t)\right|_{r}^{r}+\left\|u_{t}(t)\right\|_{0}^{2}+\|u(t)\|_{0}^{2}+\int_{0}^{t}\left\|u_{t}(s)\right\|_{0}^{2} d s \leq \Lambda_{1}(R)$;

(ii) $\left\|u_{t t}(t)\right\|_{0}^{2}+\int_{0}^{t}\left\|u_{t t}(s)\right\|_{0}^{2} d s \leq \Lambda_{2}(R)$,

for any $t>0$; here $\Lambda_{1}(R)$ and $\Lambda_{2}(R)$ are constants, which are dependent of $R$.

Proof. Multiplying (1) by $u_{t}$, integrating in $d x$ over $\Omega$, and then integrating in $d t$ on $[0, t]$, we have

$$
\left|u_{t}(t)\right|_{r}^{r}+\left\|u_{t}(t)\right\|_{0}^{2}+\|u(t)\|_{0}^{2}+\int_{0}^{t}\left\|u_{t}(s)\right\|_{0}^{2} d s \leq \Lambda_{1}(R) .
$$

Multiplying (1) by $u_{t t}$ and integrating by parts, we get

$$
\begin{aligned}
& \int_{\Omega}(r-1)\left|u_{t}\right|^{r-2}\left|u_{t t}\right|^{2}+\left\|u_{t t}\right\|_{0}^{2} \\
& \leq \int_{\Omega}|\nabla u|\left|\nabla u_{t t}\right|+\mu \int_{\Omega}\left|\nabla u_{t}\right|\left|\nabla u_{t t}\right| \\
& \quad+\int_{\Omega}|f(u)|\left|u_{t t}\right|+\int_{\Omega}|g(x)|\left|u_{t t}\right| .
\end{aligned}
$$

By Holder's and Young's inequality associated with the Sobolev embedding theorem, we find that (25) implies that

$$
\frac{1}{2}\left\|u_{t t}\right\|_{0}^{2} \leq C\|u\|_{0}^{10}+2 \mu^{2}\left\|u_{t}\right\|_{0}^{2}+C|g(x)|_{2}^{2}+C \operatorname{mes}(\Omega),
$$

where mes $(\Omega)$ denotes the measure of $\Omega$.

Multiplying (1) by $u_{t t}$ and integrating by parts, we get

$$
\begin{array}{r}
\left\|u_{t t}\right\|_{0}^{2}+\frac{d}{d t}\left[\int_{\Omega} \nabla u \nabla u_{t}+\mu \int_{\Omega}\left|\nabla u_{t}\right|^{2}\right. \\
\left.\quad+\int_{\Omega} f(u) u_{t}+\int_{\Omega} g(x) u_{t}\right] \\
\leq \int_{\Omega}\left|\nabla u_{t}\right|^{2}+\int_{\Omega} f^{\prime}(u)\left|u_{t}\right|^{2} .
\end{array}
$$

Combining with (11), we are led to the following estimation:

$$
\int_{\Omega} f^{\prime}(u)\left|u_{t}\right|^{2} \leq C\|u\|_{0}^{4}\left\|u_{t}\right\|_{0}^{2}
$$

Applying (i), estimation (ii) follows by denoting $\Lambda_{2}=$ $C\left(\Lambda_{1}+|g(x)|_{2}^{2}+\operatorname{mes}(\Omega)\right)$.

Based on the estimations above, the existence and uniqueness of the global weak solutions for (1) with the initial conditions can be obtained by standard Faedo-Galerkin method, which we omit here (see examples in Evans [17]).

Theorem 3. Let $\Omega \subset \mathbb{R}^{3}$ be a bounded domain. The assumptions (11)-(19) hold. $u_{0}(x), u_{1}(x) \in H_{0}^{1}(\Omega) . g(x) \in$ $L^{2}(\Omega)$. Then (1) have such weak solutions $u(x, t)$ : for all $T>0$

$$
u, u_{t}, u_{t t}, \in L^{\infty}\left(0, T ; H_{0}^{1}(\Omega)\right) ;
$$

for all $\varphi \in H_{0}^{1}(\Omega)$,

$$
\begin{aligned}
& \left\langle\left(\left|u_{t}\right|^{r-2} u_{t}\right)_{t}-\Delta u-\mu \Delta u_{t}-\Delta u_{t t}+f(u)-g(x), \varphi\right\rangle \\
& =0, \quad \text { a.e. } t \in[0, T] .
\end{aligned}
$$

Furthermore,

$$
\left.u\right|_{t=0}=u_{0}(x),\left.\quad u_{t}\right|_{t=0}=u_{1}(x), \quad \text { in } H_{0}^{1}(\Omega) .
$$

The weak solutions of (1) are unique and continuously dependent on initial conditions.

Lemma 4. Let $\Omega \subset \mathbb{R}^{3}$ be a bounded domain. The assumptions (11)-(19) hold. Let $z_{0}^{i}(x) \in E_{0}, i=1,2$, be two initial values, $g(x) \in L^{2}(\Omega)$, and denote by $z^{i}$ the two corresponding solutions to problem (1) in time interval $I=[0, T]$. Then the following estimate holds, for all $t \in[0, T]$,

$$
\left\|z^{1}(t)-z^{2}(t)\right\|_{E_{0}}^{2} \leq Q\left(\left\|z_{0}^{1}\right\|_{E_{0}},\left\|z_{0}^{2}\right\|_{E_{0}}\right) e^{\alpha t}\left(\left\|z_{0}^{1}-z_{0}^{2}\right\|_{E_{0}}^{2}\right),
$$

the constant $\alpha$ is only dependent on $\mu$ but independent of $T, t$, and $z_{0}^{i}(x)(i=1,2)$.

From Theorem 3 and Lemma 4, the initial boundary value problem (1) is equivalent to a continuous semigroup $\{S(t)\}_{t \geq 0}$ defined by

$$
S(t): E_{0} \longrightarrow E_{0}, \quad S(t):\left(u_{0}, u_{1}\right) \longmapsto\left(u, u_{t}\right) .
$$

\section{Bounded Absorbing Set}

We now deal with the dissipative feature of the semigroup $\{S(t)\}_{t \geq 0}$. Namely, we show that the trajectories originating from any given bounded set eventually fall, uniformly in time, into a bounded absorbing set $B_{0} \subset E_{0}$.

Theorem 5. $\{S(t)\}_{t \geq 0}$ has a bounded absorbing set $B_{0}$ in $E_{0}$; that is, for any bounded subset $B \subset E_{0}$, there exists $T_{0}=T_{0}(B)$ such that

$$
S(t) B \subset B_{0} \quad \forall t \geq T_{0} .
$$

Proof. We set $v=u_{t}+\delta u$ and rewrite (1) as follows:

$$
\begin{aligned}
& \left(\left|u_{t}\right|^{r-2} u_{t}\right)_{t}+(\mu-\delta) A v+A v_{t}+\left(1-\mu \delta+\delta^{2}\right) A u \\
& =-f(u)+g(x),
\end{aligned}
$$

and we take the $L^{2}$ inner product of (35) with $v$ and set $\delta$ small enough and

$$
\begin{aligned}
E_{1}(t)= & \frac{r-1}{r}\left|u_{t}\right|_{r}^{r}+\delta \int_{\Omega}\left|u_{t}\right|^{r-2} u_{t} u+\frac{1}{2}\|v\|_{0}^{2} \\
& +\frac{1}{2}\left(1-\mu \delta+\delta^{2}\right)\|u\|_{0}^{2}+F(u), \\
E_{2}(t)= & \delta\left(1-\mu \delta+\delta^{2}\right)\|u\|_{0}^{2}+(\mu-\delta)\|v\|_{0}^{2} \\
& +\delta \int_{\Omega} f(u) u+\delta\left|u_{t}\right|_{r}^{r}-\int_{\Omega} g(x) v
\end{aligned}
$$


then we obtain

$$
E_{1}(t)=-\int_{0}^{t} E_{2}(s) d s+2 \delta \int_{0}^{t}\left|u_{t}(s)\right|_{r}^{r} d s+E_{1}(0) .
$$

Young's inequality gives that there exist positive constants $C_{1}, C_{2}$, and $k_{1}$ only dependent on $\Omega, \delta$ such that

$$
\begin{gathered}
E_{1}(t) \geq C_{1}\left(\frac{r-1}{r}\left|u_{t}\right|_{r}^{r}+\|v\|_{0}^{2}+\omega\|u\|_{0}^{2}\right)-\frac{C_{1}}{\omega}\left|u_{t}\right|_{r}^{2(r-1)}-k_{1} \\
E_{2}(t) \geq C_{2}\left(\frac{r-1}{r}\left|u_{t}\right|_{r}^{r}+\|v\|_{0}^{2}+\omega\|u\|_{0}^{2}\right)-k_{2},
\end{gathered}
$$

here $\omega=1-\mu \delta+\delta^{2}-\left(\lambda / \lambda_{1}\right)-\left(\delta \epsilon / \lambda_{1}\right)>0$ as $\delta$ small enough and $k_{2}=\left(|g|_{2}\right)$.

By Lemma 2, this shows that

$$
\begin{aligned}
C_{1}( & \left.\frac{r-1}{r}\left|u_{t}\right|_{r}^{r}+\|v\|_{0}^{2}+\Phi\|u\|_{0}^{2}\right)-k_{1} \\
\leq & -\int_{0}^{t} C_{2}\left(\frac{r-1}{r}\left|u_{t}\right|_{r}^{r}+\|v\|_{0}^{2}+\Phi\|u\|_{0}^{2}-\frac{k_{2}}{C_{2}}\right) \\
& +2 \delta \int_{0}^{t}\left|u_{t}(s)\right|_{r}^{r} d s+C_{1} \frac{1}{\omega}\left|u_{t}\right|_{r}^{2(r-1)}+E_{1}(0) \\
\leq & -\int_{0}^{t} C_{2}\left(\frac{r-1}{r}\left|u_{t}\right|_{r}^{r}+\|v\|_{0}^{2}+\Phi\|u\|_{0}^{2}-\frac{k_{2}}{C_{2}}\right) \\
& +\Lambda(R)^{(r-2) / r}\left(2 \delta \int_{0}^{t}\left|u_{t}(s)\right|_{r}^{2} d s+C_{1} \frac{1}{\omega}\left|u_{t}\right|_{r}^{r}\right)+E_{1}(0)
\end{aligned}
$$

Then we get

$$
\begin{aligned}
C_{1}\left(\frac{r-1}{r}\left|u_{t}\right|_{r}^{r}+\|v\|_{0}^{2}+\Phi\|u\|_{0}^{2}\right)-k_{1} \\
\leq-\int_{0}^{t} C_{2}\left(\frac{r-1}{r}\left|u_{t}\right|_{r}^{r}+\|v\|_{0}^{2}+\omega\|u\|_{0}^{2}-\frac{k_{2}}{C_{2}}\right) \\
\quad+\theta \Lambda(R)+E_{1}(0),
\end{aligned}
$$

for any $M>k_{2} / C_{2}$ and $\theta=\max \left\{2 \delta, C_{1} / \omega\right\}$.

Hence, there exists $T_{0}=T_{0}(B)$ such that

$$
\frac{r-1}{r}\left|u_{t}\left(T_{0}\right)\right|_{r}^{r}+\left\|v\left(T_{0}\right)\right\|_{0}^{2}+\omega\left\|u\left(T_{0}\right)\right\|_{0}^{2} \leq M .
$$

According to Lemma 2, it follows that if $t \geq T_{0}(B)$, then there exists $M>0$, such that

$$
\left|u_{t}\right|_{r}^{r}+\|v\|_{0}^{2}+\|u\|_{0}^{2} \leq M,
$$

which completes the proof of the desired results.

Corollary 6. For any $B \subset E_{0}$, there exists $T=T(B)$, such that

$$
\int_{s}^{t}\left(\left\|u_{t}(\tau)\right\|_{0}^{2}+\left\|u_{t t}(\tau)\right\|_{0}^{2}\right) d \tau+\left\|u_{t}(t)\right\|_{0}^{2}+\left\|u_{t t}(t)\right\|_{0}^{2} \leq M_{1}
$$

provided that $t \geq s \geq T$, where $M_{1}$ is a positive constant only dependent on $C$ (given by (16) and (18)) and $M$ (given by (42)).
Proof. Multiplying (1) by $u_{t}(t), u_{t t}$, respectively, integrating in $d x$ over $\Omega$, and then integrating in $d t$ on $[s, t]$ and associated with Theorem 5 and Sobolev embedding $H_{0}^{1} \hookrightarrow L^{r}$, we can get the conclusions above.

Hereafter, we always assume that for some $\delta>0$ and $v=$ $u_{t}+\delta u$

$$
B_{0}=\left\{(u, v) \in E_{0} ;\|u\|+\|v\| \leq M\right\}
$$

is the bounded absorbing set of $\{S(t)\}_{t \geq 0}$ in $E_{0}$ obtained in Theorem 5.

\section{Asymptotic Regularity of the Solutions}

In this section, we will establish some a priori estimates about the solutions of (1), which are the basis of our analysis. Let $u(x, t)$ be a unique weak solution of (1) corresponding to the initial data $z_{0}=\left(u_{0}, u_{1}\right) \in H_{0}^{1}(\Omega) \times H_{0}^{1}(\Omega)$. We decompose $u$ into the sum

$$
u(t)=v(t)+w(t)
$$

where $v(t)$ and $w(t)$ are the solutions to the problems

$$
\begin{gathered}
\left(\left|v_{t}\right|^{r-2} v_{t}\right)_{t}-\Delta v-\mu \Delta v_{t}-\Delta v_{t t}+f_{0}(v)=0, \\
\left.v\right|_{\partial \Omega}=0, \\
v(0)=u_{0}, \quad v_{t}(0)=u_{1}, \\
\left(\left|u_{t}\right|^{r-2} u_{t}-\left|v_{t}\right|^{r-2} v_{t}\right)_{t}-\Delta w-\mu \Delta w_{t} \\
-\Delta w_{t t}+f(u)-f_{0}(v)=g, \\
\left.w\right|_{\partial \Omega}=0, \\
w(0)=0, \quad w_{t}(0)=0 .
\end{gathered}
$$

It is convenient to denote

$$
\begin{gathered}
z(t)=\left(u(t), u_{t}(t)\right), \quad z_{d}(t)=\left(v(t), v_{t}(t)\right) \\
z_{c}(t)=\left(w(t), w_{t}(t)\right) .
\end{gathered}
$$

Lemma 7. For any $R \geq 0$, there exist $M_{2}=M_{2}(R) \geq 0$ and $k_{0}>0$, such that whenever $\left\|z_{0}\right\|_{0} \leq R$, it follows that

$$
\left\|z_{d}(t)\right\|_{0} \leq M_{2} e^{-k_{0} t}, \quad \forall t \in \mathbb{R}^{+},
$$

the constant $k_{0}$ is independent of $R$ and $t$.

Proof. Denoting $\xi=v_{t}+\delta v$ and repeating word by word Theorem 5 , that applies to the present case with $z_{d}(t)$ in place of $z(t)$ (with the further simplification that $C=0$ for now $\left.f_{1}=0, g=0\right)$.

Set

$$
E(t)=\frac{1}{2}\|\xi\|_{0}^{2}+\frac{1}{2}\left(1-\mu \delta+\delta^{2}\right)\|v\|_{0}^{2}+F_{0}(v) .
$$


Let $\delta \leq \min (\mu / 2,1 / \mu)$ be small enough and $C_{\delta}=$ $\left(\frac{1}{2}\right) \max \left(1,1-\mu \delta+\delta^{2}\right)>0$; then

$$
E(t) \geq C_{\delta}\left(\|v\|_{0}^{2}+\|\xi\|_{0}^{2}\right) .
$$

Combining with (13) we get the differential inequality

$$
\begin{aligned}
& \frac{d}{d t} E(t)+\delta\left(1-\mu \delta+\delta^{2}\right)\|v\|_{0}^{2}+(\mu-\delta)\|\xi\|_{0}^{2} \\
& \quad \leq(r-1) \kappa_{r}^{2}\left|v_{t}\right|_{r}^{(r-2)}\left\|v_{t t}\right\|_{0}\|\xi\|_{0}-\delta \int_{\Omega} f_{0}(v) v \\
& \quad \leq \frac{(r-1)^{2} \kappa_{r}^{4}}{2(\mu-\delta)}\left|v_{t}\right|_{r}^{2(r-2)}\left\|v_{t t}\right\|_{0}^{2}+\frac{\mu-\delta}{2}\|\xi\|_{0}^{2} \\
& \quad \leq C_{\mu}\left|v_{t}\right|_{r}^{2(r-3)}\left\|v_{t t}\right\|_{0}^{2}\left[\|\xi\|_{0}^{2}+\|v\|_{0}^{2}\right]+\frac{\mu-\delta}{2}\|\xi\|_{0}^{2},
\end{aligned}
$$

where $C_{\mu}=(r-1)^{2} \kappa_{r}^{5} / 2(\mu-\delta)$ and $\kappa_{r}$ is the Sobolev embedded constant $H_{0}^{1} \hookrightarrow L^{r}$.

Multiplying (46) by $v_{t}(t)$, integrating in $d x$ over $\Omega$, and then integrating in $d \tau$ on $[0, t]$,

$$
\sup _{\left\|z_{0}\right\|_{0} \leq R} \sup _{t \in \mathbb{R}^{+}}\left\|z_{d}(t)\right\|_{0}<+\infty .
$$

So we get

$$
\int_{\Omega} F_{0}(v) \leq C\left(|v|^{2}+|v|_{6}^{6}\right) \leq k\|v\|_{0}^{2}, \quad \forall t \geq 0,
$$

where $k=k(R) \geq 1$.

By (50),

$$
\begin{aligned}
E(t) & \leq \frac{1}{2}\|\xi\|_{0}^{2}+\frac{1}{2}\left(1+k-\mu \delta+\delta^{2}\right)\|v\|_{0}^{2} \\
& \leq \frac{1}{2}\left(1+k-\mu \delta+\delta^{2}\right)\left(\|\xi\|_{0}^{2}+\|v\|_{0}^{2}\right),
\end{aligned}
$$

and by the above

$$
\delta\left(1-\mu \delta+\delta^{2}\right)\|v\|_{0}^{2}+\frac{1}{2}(\mu-\delta)\|\xi\|_{0}^{2} \geq \alpha\left(\|\xi\|_{0}^{2}+\|v\|_{0}^{2}\right),
$$

here $\alpha=\min \left\{\delta\left(1-\mu \delta+\delta^{2}\right),(1 / 2)(\mu-\delta)\right\}$. Then there exists a constant $k_{0}\left(=2 \alpha /\left(1+k-\mu \delta+\delta^{2}\right)\right)$ such that

$$
k_{0} E(t) \leq \delta\left(1-\mu \delta+\delta^{2}\right)\|v\|_{0}^{2}+\frac{1}{2}(\mu-\delta)\|\xi\|_{0}^{2} .
$$

Combining (51), (57), and Young's inequality, we are led to the differential inequality

$$
\frac{d}{d t} E(t) \leq\left(-k_{0}+\frac{C_{\mu}}{C_{\delta}}\left|v_{t}\right|_{r}^{2(r-3)}\left\|v_{t t}\right\|_{0}^{2}\right) E(t) .
$$

By Gronwall Lemma, we get

$$
E(t) \leq E(0) e^{-k_{0} t} e^{\int_{0}^{t}\left(C_{\mu} / C_{\delta}\right)\left|v_{t}(s)\right|_{r}^{2(r-3)}\left\|v_{t t}(s)\right\|_{0}^{2} d s} \quad \forall t \geq 0 .
$$

Due to Lemma 2 and (46), then $\left\|z_{d}(0)\right\|_{0}=\left\|z_{0}\right\|<R$; for any $t>0$, there holds

$$
E(t) \leq E(0) e^{\left(C_{\mu} / C_{\delta}\right) \Lambda_{1}^{2(r-3) / r} \Lambda_{2}} e^{-k_{0} t} \quad \forall t \geq 0 .
$$

From (57) we get

$$
E(0) \leq \frac{\omega}{\omega}\left\|z_{0}\right\|_{0}^{2}
$$

where $\omega=\min \left\{\delta\left(1-\mu \delta+\delta^{2}\right),(1 / 2)(\mu-\delta)\right\}$. Putting together (51), the proof is finished.

Lemma 8. For any $R \geq 0$, there exist $M_{3}=M_{3}(R) \geq 0$ and $k_{1}=k_{1}(R)>0$, such that whenever $\left\|z_{0}\right\|_{0} \leq R$ and for any time $t \in \mathbb{R}^{+}$and every $\mu$, the solutions of (47) satisfy the following estimates:

$$
\begin{aligned}
\left\|z_{c}(t)\right\|_{\sigma}^{2} & =\left|A^{(1+\sigma) / 2} w(t)\right|_{2}^{2}+\left|A^{(1+\sigma) / 2} w_{t}(t)\right|_{2}^{2} \\
& \leq M_{3}\left(1+|g|_{2}^{2}\right) e^{k_{1} t}
\end{aligned}
$$

Proof. Due to Lemma 2, Theorem 5, and Corollary 6, there exists an increasing function $Q_{1}(\cdot)$ in $[0,+\infty)$, such that

$$
\begin{aligned}
\left|u_{t}\right|_{r}^{r} & +\left|v_{t}\right|_{r}^{r}+\left\|u_{t t}\right\|_{0}^{2}+\left\|v_{t t}\right\|_{0}^{2}+\|u\|_{0}^{2}+\|v\|_{0}^{2} \\
& \leq Q_{1}(R), \quad \forall t \in \mathbb{R}^{+} .
\end{aligned}
$$

Choosing

$$
\sigma=\min \left\{\frac{1}{4}, \frac{5-p}{2}\right\}
$$

and multiplying (47) by $A^{\sigma} w_{t}$, we are led to the identity

$$
\begin{aligned}
\frac{1}{2} \frac{d}{d t}\left\|z_{c}\right\|_{\sigma}^{2}+\mu\left\|A^{(1+\sigma) / 2} w_{t}\right\|^{2} \\
=-\left\langle\left(\left|u_{t}\right|^{r-2} u_{t}-\left|v_{t}\right|^{r-2} v_{t}\right)_{t}, A^{\sigma} w_{t}\right\rangle \\
\quad-\left\langle f(u)-f(v), A^{\sigma} w_{t}\right\rangle-\left\langle f_{1}(v), A^{\sigma} w_{t}\right\rangle+\left\langle g, A^{\sigma} w_{t}\right\rangle
\end{aligned}
$$

where

$$
\left\|z_{c}\right\|_{\sigma}^{2}=\left|A^{(1+\sigma) / 2} w\right|_{2}^{2}+\left|A^{(1+\sigma) / 2} w_{t}\right|_{2}^{2} .
$$

Combining with $(r-2) /(4-2 \sigma) \leq 1$ and (2), we have

$$
\begin{aligned}
& \left\langle\left(\left|u_{t}\right|^{r-2} u_{t}-\left|v_{t}\right|^{r-2} v_{t}\right)_{t}, A^{\sigma} w_{t}\right\rangle \\
& \leq(r-1) \int_{\Omega}\left(\left|u_{t}\right|^{r-2}\left|u_{t t}\right|+\left|v_{t}\right|^{r-2}\left|v_{t t}\right|\right)\left|A^{\sigma} w_{t}\right| \\
& \quad \leq C\left(\left|u_{t}\right|_{6(r-2) /(4-2 \sigma)}^{r-2}\left|u_{t t}\right|_{6}+\left|v_{t}\right|_{6(r-2) /(4-2 \sigma)}^{r-2}\left|v_{t t}\right|_{6}\right)\left|A^{\sigma} w_{t}\right|_{6 /(1+2 \sigma)} \\
& \leq C\left(\left\|u_{t}\right\|_{0}^{(r-2)}\left\|u_{t t}\right\|_{0}+\left\|v_{t}\right\|_{0}^{(r-2)}\left\|v_{t t}\right\|_{0}\right)\left|A^{(1+\sigma) / 2} w_{t}\right|_{2} \\
& \quad \leq C Q_{1}(R)^{2(r-1)}+\frac{\mu}{4}\left|A^{(1+\sigma) / 2} w_{t}\right|_{2}^{2} .
\end{aligned}
$$


By virtue of (11), we get

$$
\begin{aligned}
& -\left\langle f(u)-f(v), A^{\sigma} w_{t}\right\rangle \\
& \quad \leq C \int_{\Omega}\left(1+|u|^{4}+|v|^{4}\right)|w|\left|A^{\sigma} w_{t}\right| \\
& \leq C\left(1+|u|_{6}^{4}+|v|_{6}^{4}\right)|w|_{6 /(1-2 \sigma)}\left|A^{\sigma} w_{t}\right|_{6 /(1+2 \sigma)} \\
& \quad \leq C\left(1+\|u\|_{0}^{4}+\|v\|_{0}^{4}\right)\left\|A^{(1+\sigma) / 2} w\right\|\left\|A^{(1+\sigma) / 2} w_{t}\right\| \\
& \quad \leq C\left(1+Q_{1}(R)^{4}\right)\left\|z_{c}\right\|_{\sigma}^{2}+\frac{\mu}{4}\left\|A^{(1+\sigma) / 2} w_{t}\right\|^{2} .
\end{aligned}
$$

Since $p /(5-2 \sigma) \leq 1$, by (14) we deduce that

$$
\begin{aligned}
\left\langle f_{1}(v), A^{\sigma} w_{t}\right\rangle & \leq C \int_{\Omega}\left(1+|v|^{p}\right)\left|A^{\sigma} w_{t}\right| \\
& \leq C\left(1+|v|_{6 p /(5-2 \sigma)}^{p}\right)\left|A^{\sigma} w_{t}\right|_{6 /(1+2 \sigma)} \\
& \leq C\left(1+\|v\|_{0}^{p}\right)\left\|A^{(1+\sigma) / 2} w_{t}\right\| \\
& \leq C\left(1+Q_{1}(R)^{p}\right)+\frac{\mu}{4}\left\|A^{(1+\sigma) / 2} w_{t}\right\|^{2} .
\end{aligned}
$$

Finally,

$$
\begin{aligned}
\left\langle g, A^{\sigma} w_{t}\right\rangle & \leq C\left\|A^{-1 / 2} g\right\|\left\|A^{(1+\sigma) / 2} w_{t}\right\| \\
& \leq C|g|_{2}^{2}+\frac{\mu}{4}\left\|A^{(1+\sigma) / 2} w_{t}\right\|^{2} .
\end{aligned}
$$

Plugging (67)-(70) into (65), we obtain

$$
\frac{d}{d t}\left\|z_{c}\right\|_{\sigma}^{2} \leq C\left(1+Q_{1}(R)^{4}\right)\left\|z_{c}\right\|_{\sigma}^{2}+C|g|_{2}^{2}+Q_{2}(R)
$$

where $Q_{2}(\cdot)$ is an increasing function in $[0,+\infty)$ and the Gronwall lemma entails, there exists a constant $M_{3}=M_{3}(R)$ such that

$$
\left\|z_{c}\right\|_{\sigma}^{2} \leq M_{3}(R)\left(1+|g|_{2}^{2}\right) e^{k_{1} t} \quad \forall t \in[0, T] .
$$

The proof is finished.

Lemma 9. Let $\Omega \subset \mathbb{R}^{3}$ be a bounded domain. The assumptions (11)-(15) hold, and let $\left(u(t), u_{t}(t)\right)$ be the solution of (1) corresponding to the initial data $z_{0}=\left(u_{0}, u_{1}\right)$. Then, for any $\gamma>0$, one can decompose $u(t)$ as

$$
u(t)=v_{1}(t)+w_{1}(t) \quad \forall t \geq 0,
$$

where $v_{1}(t)$ and $w_{1}(t)$ satisfy the following estimates: for all $t \geq$ 0 ,

$$
\begin{gathered}
\int_{s}^{t}\left\|v_{1}(\tau)\right\|_{0}^{2} d \tau \leq \gamma(t-s)+C_{\gamma} \\
\left|A^{(1+\sigma) / 2} w_{1}(t)\right|_{2}^{2} \leq K_{\gamma}
\end{gathered}
$$

with the constants $C_{\varepsilon}$ and $K_{\gamma}$ depending on $\gamma,\left\|z_{0}\right\|_{0}$, and $|g|_{2}^{2}$ but both being independent of $t$.

Proof. From Theorem 5, we know that there exists a constant $M_{\left\|z_{0}\right\|_{0}}$ which depends only on the $E_{0}$-bound of $z_{0}$, such that

$$
\sup _{t \geq 0}\left\|S(t) z_{0}\right\|_{0}^{2} \leq M_{\left\|z_{0}\right\|_{0}} .
$$

Now, taking $T \geq\left(1 / k_{0}\right) \ln (\beta E(0) / \varepsilon)$ and in every interval $[m T,(m+1) T), m=1,2, \ldots$, we set

$$
v_{1}(t)=v(t), \quad w_{1}(t)=w(t),
$$

where $v(t)$ and $w(t)$ are the solutions of (46) and (47), respectively, in the interval $[(m-1) T,(m+1) T)$ with the initial data $\left(v((m-1) T), v_{t}((m-1) T)\right)=S(t)((m-1) T) z_{0}$ and $\left(w((m-1) T), w_{t}((m-1) T)\right)=(0,0,0)$. And in interval $[0, T)$, we set $v_{1}(t)=v(t)$ and $w_{1}(t)=w(t)$, where $v(t)$ and $w(t)$ are the solutions of (46) and (47), respectively, in the interval $[0, T)$ with the initial data $\left(v(0), v_{t}(0)\right)=z_{0}$ and $\left(w(0), w_{t}(0)\right)=(0,0)$.

Then from Lemma 7, we have

$$
\int_{s}^{t}\left\|v_{1}(\tau)\right\|_{0}^{2} d \tau \leq \gamma(t-s)+\chi_{[0, T)} M_{2} \quad \forall 0 \leq s \leq t,
$$

and from Lemma 8, we have

$$
\left|A^{(1+\sigma) / 2} w_{1}(t)\right|_{2}^{2} \leq M_{3}(R)\left(1+|g|_{2}^{2}\right) e^{2 k_{1} T} \quad \forall t \geq 0
$$

where $\chi_{[0, T)}$ is the characteristic function of set $[0, T)$.

Remark 10. From the proof of Lemma 9, we observe that the decomposition $v_{1}(t)$ and $w_{1}(t)$ also satisfy further that

$$
\left\|v_{1}(t)\right\|_{0}^{2} \leq M_{0}(R) \quad \forall t \geq 0 .
$$

In what follows we begin to establish the asymptotic regularity of the solutions. Now we can claim the following result.

Lemma 11. Let $\Omega \subset \mathbb{R}^{3}$ be a bounded domain with smooth boundary, and assume that $f$ satisfies (11)-(15); there exists constant $\mathscr{J}_{0}$ which depends only on the $E_{0}$-bounds of $B\left(\subset E_{0}\right)$, such that for any $t \geq 0$ and $z_{0} \in B$

$$
\left\|z_{c}(t)\right\|_{\sigma}^{2} \leq \mathscr{J}_{0}
$$

holds.

Proof. Let $\epsilon=\min (1 / 4, \mu / 2)$ and multiplying (47) by $A^{\sigma}\left(w_{t}(t)+\epsilon w(t)\right)$, we get that

$$
\begin{aligned}
\frac{1}{2} \frac{d}{d t}[ & \left.(1+\mu \epsilon)\left|A^{(1+\sigma) / 2} w\right|_{2}^{2}+\left|A^{(1+\sigma) / 2} w_{t}\right|_{2}^{2}+2\left\langle-\Delta w_{t}, \epsilon A^{\sigma} w\right\rangle\right] \\
+ & (\mu-\epsilon)\left|A^{(1+\sigma) / 2} w_{t}\right|_{2}^{2}+\epsilon\left|A^{(1+\sigma) / 2} w\right|_{2}^{2} \\
= & -\left\langle\left(\left|u_{t}\right|^{r-2} u_{t}-\left|v_{t}\right|^{r-2} v_{t}\right)_{t}, A^{\sigma}\left(w_{t}+\epsilon w\right)\right\rangle \\
& -\left\langle f(u)-f(v), A^{\sigma}\left(w_{t}+\epsilon w\right)\right\rangle-\left\langle f_{1}(v), A^{\sigma}\left(w_{t}+\epsilon w\right)\right\rangle \\
& +\left\langle g, A^{\sigma}\left(w_{t}+\epsilon w\right)\right\rangle .
\end{aligned}
$$


Let

$$
\begin{aligned}
H(t)= & (1+\mu \epsilon)\left|A^{(1+\sigma) / 2} w\right|_{2}^{2} \\
& +\left|A^{(1+\sigma) / 2} w\right|_{2}^{2}+2\left\langle-\Delta w_{t}, \epsilon A^{\sigma} w\right\rangle,
\end{aligned}
$$

then

$$
H(t) \leq \omega_{1}\left(\left|A^{(1+\sigma) / 2} w\right|_{2}^{2}+\left|A^{(1+\sigma) / 2} w\right|_{2}^{2}\right)
$$

here $\Phi_{1}=\max (1+(1+\mu) \epsilon, 1+\epsilon)>1$ and

$$
H(t) \geq \omega_{2}\left(\left|A^{(1+\sigma) / 2} w\right|_{2}^{2}+\left|A^{(1+\sigma) / 2} w\right|_{2}^{2}\right)
$$

where $\omega_{2}=\min (1+(\mu-1) \epsilon, 1-\epsilon)$.

Combining with (2), (63), and $(r-2) /(4-2 \sigma) \leq 1$, we have

$$
\begin{aligned}
&\left\langle\left(\left|u_{t}\right|^{r-2} u_{t}-\left|v_{t}\right|^{r-2} v_{t}\right)_{t}, A^{\sigma}\left(w_{t}+\epsilon w\right)\right\rangle \\
& \leq(r-1) \int_{\Omega}\left(\left|u_{t}\right|^{r-2}\left|u_{t t}\right|+\left|v_{t}\right|^{r-2}\left|v_{t t}\right|\right)\left(\left|A^{\sigma} w_{t}\right|+\epsilon\left|A^{\sigma} w\right|\right) \\
& \leq C\left(\left|u_{t}\right|_{6(r-2) /(4-2 \sigma)}^{r-2}\left|u_{t t}\right|_{6}+\left|v_{t}\right|_{6(r-2) /(4-2 \sigma)}^{r-2}\left|v_{t t}\right|_{6}\right) \\
& \times\left(\left|A^{\sigma} w_{t}\right|_{6 /(1+2 \sigma)}+\epsilon\left|A^{\sigma} w\right|_{6 /(1+2 \sigma)}\right) \\
& \leq C\left(\left\|u_{t}\right\|_{0}^{(r-2)}\left\|u_{t t}\right\|_{0}+\left\|v_{t}\right\|_{0}^{(r-2)}\left\|v_{t t}\right\|_{0}\right) \\
& \times\left(\left|A^{(1+\sigma) / 2} w_{t}\right|_{2}+\epsilon\left|A^{(1+\sigma) / 2} w\right|_{2}\right) \\
& \leq C Q_{1}(R)^{2(r-1)}+\frac{\mu-\epsilon}{8}\left|A^{(1+\sigma) / 2} w_{t}\right|_{2}^{2}+\frac{\epsilon}{8}\left|A^{(1+\sigma) / 2} w\right|_{2}^{2} .
\end{aligned}
$$

Using Lemma 8 to deal with the nonlinear term, we get

$$
\begin{gathered}
-\left\langle f(u)-f(v), A^{\sigma} w_{t}\right\rangle \leq C \int_{\Omega}\left(1+|u|^{4}+|v|^{4}\right)|w|\left|A^{\sigma} w_{t}\right|, \\
\int_{\Omega}|u|^{4}|w|\left|A^{\sigma} w_{t}\right| \leq \int_{\Omega}\left(\left|v_{1}\right|^{4}+\left|w_{1}\right|^{4}\right)|w|\left|A^{\sigma} w_{t}\right|
\end{gathered}
$$

Using Remark 10, we have

$$
\begin{aligned}
\int_{\Omega}\left|v_{1}\right|^{4}|w|\left|A^{\sigma} w_{t}\right| \leq & C\left(\left|v_{1}\right|_{6}^{4}\right)|w|_{6 /(1-2 \sigma)}\left|A^{\sigma} w_{t}\right|_{6 /(1+2 \sigma)} \\
\leq & C\left\|v_{1}\right\|_{0}^{4}\left|A^{(1+\sigma) / 2} w\right|_{2}\left|A^{(1+\sigma) / 2} w_{t}\right|_{2} \\
\leq & C M_{0}(R)^{2}\left\|v_{1}\right\|_{0}^{2} \\
& \times\left(\left|A^{(1+\sigma) / 2} w\right|_{2}^{2}+\left|A^{(1+\sigma) / 2} w_{t}\right|_{2}^{2}\right) .
\end{aligned}
$$

Moreover, from Lemma 2, Remark 10, and Lemma 8, there exists a constant $M_{4}=M_{4}(R)$ such that, for all $t \geq 0$,

$$
\begin{aligned}
& \left\|z_{c}(t)\right\|_{0}^{2}=\|w(t)\|_{0}^{2}+\left\|w_{t}(t)\right\|_{0}^{2} \leq M_{4}, \\
& \int_{\Omega}\left|w_{1}\right|^{4}|w|\left|A^{\sigma} w_{t}\right| \\
& \leq C\left|w_{1}\right|_{6 /(1-2 \sigma)}^{4}|w|_{6 /(1+6 \sigma)}\left|A^{\sigma} w_{t}\right|_{6 /(1+2 \sigma)} \\
& \leq C\|w\|_{0}^{2}\left|A^{(1+\sigma) / 2} w_{1}\right|_{2}^{4}\left|A^{(1+\sigma) / 2} w_{t}\right|_{2} \\
& \leq C M_{4} K_{\gamma}^{2}\left|A^{(1+\sigma) / 2} w_{t}\right|_{2} \\
& \leq C M_{4}^{2} K_{\gamma}^{4}+\frac{\mu-\epsilon}{8}\left|A^{(1+\sigma) / 2} w_{t}\right|_{2}^{2}
\end{aligned}
$$

where $K_{\gamma}$ is given in (75).

At the same time, we get

$$
\int_{\Omega}|v|^{4}|w|\left|A^{\sigma} w_{t}\right| \leq C_{M}\|v\|_{0}^{4}\left(\left|A^{(1+\sigma) / 2} w\right|_{2}^{2}+\left|A^{(1+\sigma) / 2} w_{t}\right|_{2}^{2}\right) .
$$

Similarly

$$
\begin{aligned}
&-\left\langle f(u)-f(v), A^{\sigma} w\right\rangle \\
& \leq C M_{0}(R)^{2}\left\|v_{1}\right\|_{0}^{2}\left|A^{(1+\sigma) / 2} w\right|_{2}^{2}+C\left(1+M_{4}^{2} K_{\gamma}^{4}\right) \\
&+\frac{\epsilon}{8}\left|A^{(1+\sigma) / 2} w\right|_{2}^{2}+C_{M}\|v\|_{0}^{4}\left|A^{(1+\sigma) / 2} w\right|_{2}^{2} .
\end{aligned}
$$

Since $p /(5-2 \sigma) \leq 1$, by (14) and Lemma 7 , we deduce that

$$
\begin{aligned}
& \left\langle f_{1}(v), A^{\sigma}\left(w_{t}+\epsilon w\right)\right\rangle \\
& \leq C \int_{\Omega}\left(1+|v|^{p}\right)\left(\left|A^{\sigma} w_{t}\right|+\epsilon|w|\right) \\
& \leq C\left(1+|v|_{6 p /(5-2 \sigma)}^{p}\right)\left(\left|A^{\sigma} w_{t}\right|_{6 /(1+2 \sigma)}+\epsilon\left|A^{\sigma} w_{t}\right|_{\sigma /(1+2 \sigma)}\right) \\
& \leq C\left(1+\|v\|_{0}^{p}\right)\left(\left|A^{(1+\sigma) / 2} w_{t}\right|_{2}+\epsilon\left|A^{(1+\sigma) / 2} w\right|_{2}\right) \\
& \quad \leq C\left(1+M_{2}^{p}\right)+\frac{\mu-\epsilon}{8}\left|A^{(1+\sigma) / 2} w_{t}\right|_{2}^{2}+\frac{\epsilon}{8}\left|A^{(1+\sigma) / 2} w\right|_{2}^{2} .
\end{aligned}
$$

Finally,

$$
\begin{aligned}
\left\langle g, A^{\sigma}\left(w_{t}+\varepsilon w\right)\right\rangle & \leq C\left|A^{-1 / 2} g\right|_{2}\left|A^{(1+\sigma) / 2}\left(w_{t}+\varepsilon w\right)\right|_{2} \\
& \leq C|g|_{2}^{2}+\frac{\mu-\epsilon}{8}\left|A^{(1+\sigma) / 2} w_{t}\right|_{2}^{2}+\frac{\epsilon}{8}\left|A^{(1+\sigma) / 2} w\right|_{2}^{2}
\end{aligned}
$$

Moreover, following Lemma 7, we can take $T_{1}$ large enough such that for all $t \geq T_{1}$

$$
\|v(t)\|_{0}^{4} \leq \min \left(\frac{\epsilon}{16 C_{M}}, \frac{\mu-\epsilon}{16 C_{M}}\right) .
$$


Plugging (86)-(93) into (65), we obtain

$$
\frac{d}{d t} H(t)+\vartheta H(t) \leq C_{R}\left\|v_{1}\right\|^{2} H(t)+C|g|_{2}^{2}+Q_{2}(R),
$$

where

$$
\vartheta=\min (\mu-\epsilon, \epsilon), \quad C_{R}=\frac{2 C M_{0}}{\omega_{2}},
$$

and $Q_{2}(\cdot)=C\left(1+Q_{1}^{p}+M_{4}^{2} K_{\gamma}^{4}\right)$ is an increasing function in $[0,+\infty)$.

Then using the Gronwall inequality and integrating over $\left[T_{1}, t\right]$, we entail

$$
\begin{aligned}
H(t) \leq & e^{-\int_{T_{1}}^{t}\left(\vartheta-C_{R}\left\|v_{1}(s)\right\|_{0}^{2}\right) d s} H\left(T_{1}\right) \\
& +\left(C|g|_{2}^{2}+Q_{2}(R)\right) \int_{T_{1}}^{t} e^{\int_{t}^{s}\left(9-C_{R}\left\|v_{1}(\tau)\right\|_{0}^{2}\right) d \tau} d s .
\end{aligned}
$$

Letting $\gamma<\vartheta / 2 C_{R}$, then we get

$$
H(t) \leq e^{-(\vartheta / 2)\left(t-T_{1}\right)} H\left(T_{1}\right)+\frac{2}{\vartheta}\left(C|g|_{2}^{2}+Q_{2}(R)\right) .
$$

Hence, by Lemma $8,(85)$ and noting that $T_{1}$ is fixed, the proof is finished.

Lemma 12. Assume that $B_{\sigma}$ is bounded in $E_{\sigma}$. Then there exists a constant $M_{\sigma}(>0)$ which depends only on the $E_{\sigma}$-bounds of $B_{\sigma}$ such that, for any $t \geq 0$ and $z_{0} \in H_{\sigma}$

$$
\|z(t)\|_{\sigma} \leq M_{\sigma}
$$

Proof. We set $v=u_{t}+\delta u$, take the $L^{2}$ inner product of (35) with $A^{\sigma} v$, and set $\delta \leq \min (\mu / 2,1 / \mu)$; we have

$$
\begin{aligned}
\frac{1}{2} \frac{d}{d t} & {\left[\left|A^{(1+\sigma) / 2} v\right|_{2}^{2}+\left(1-\mu \delta+\delta^{2}\right)\left|A^{(1+\sigma) / 2} u\right|_{2}^{2}\right] } \\
+ & (\mu-\delta)\left|A^{(1+\sigma) / 2} v\right|_{2}^{2}+\delta\left(1-\mu \delta+\delta^{2}\right)\left|A^{(1+\sigma) / 2} u\right|_{2}^{2} \\
& =-(r-1)\left\langle\left|u_{t}\right|^{r-2} u_{t t}, A^{\sigma} v\right\rangle-\left\langle f(u), A^{\sigma} v\right\rangle+\left\langle g, A^{\sigma} v\right\rangle
\end{aligned}
$$

We only need to deal with the nonlinear term separately, and the remainders are same as in Lemma 11:

$$
\begin{gathered}
-(r-1)\left\langle\left|u_{t}\right|^{r-2} u_{t t}, A^{\sigma} v\right\rangle \\
\leq(r-1)\left|u_{t}\right|_{6(r-2) /(4-2 \sigma)}^{r-2}\left|u_{t t}\right|_{6}\left|A^{\sigma} v\right|_{6 /(1+2 \sigma)} \\
\leq C\left\|u_{t}\right\|_{0}^{2(r-2)}\left\|u_{t t}\right\|_{0}^{2}+\frac{\mu-\delta}{8}\left|A^{(1+\sigma) / 2} v\right|_{2} \\
\left\langle g, A^{\sigma} v\right\rangle \leq C|g(x)|_{2}^{2}+\frac{\mu-\delta}{8}\left|A^{(1+\sigma) / 2} v\right|_{2} \\
-\left\langle f(u), A^{\sigma} v\right\rangle \leq C \int_{\Omega}\left(1+|u|^{5}\right)\left|A^{\sigma} v\right| \\
\leq C\left(1+\|v\|_{0}^{2}\right)+C \int_{\Omega}|u|^{4}|u|\left|A^{\sigma} v\right|
\end{gathered}
$$

Using Lemma 9 and Remark 10, we have

$$
\begin{gathered}
C \int_{\Omega}|u|^{4}|u|\left|A^{\sigma} v\right| \leq C \int_{\Omega}\left(\left|v_{1}\right|^{4}+\left|w_{1}\right|^{4}\right)|u|\left|A^{\sigma} v\right| \\
C \int_{\Omega}\left|v_{1}\right|^{4}|u|\left|A^{\sigma} v\right| \\
\leq C\left|v_{1}\right|_{6}^{4}|u|_{6 /(1-2 \sigma)}\left|A^{\sigma} v\right|_{6 /(1+2 \sigma)} \\
\leq C\left\|v_{1}\right\|_{0}^{4}\left|A^{(1+\sigma) / 2} u\right|_{2}\left|A^{(1+\sigma) / 2} v\right|_{2} \\
\leq C\left\|v_{1}\right\|_{0}^{2}\left[\left|A^{(1+\sigma) / 2} u\right|_{2}^{2}+\left(1-\mu \delta+\delta^{2}\right)\left|A^{(1+\sigma) / 2} v\right|_{2}^{2}\right] \\
C \int_{\Omega}\left|w_{1}\right|^{4}|u|\left|A^{\sigma} v\right| \\
\leq C|u|_{6}\left|w_{1}\right|_{6 /(1-2 \sigma)}^{4}\left|A^{\sigma} v\right|_{6 /(1+2 \sigma)} \\
\leq C\|u\|_{0}\left|A^{(1+\sigma) / 2} w_{1}\right|_{2}^{4}\left|A^{(1+\sigma) / 2} v\right|_{2} \\
\leq C\left|A^{(1+\sigma) / 2} v\right|_{2} \\
\leq C+\frac{\mu-\delta}{4}\left|A^{(1+\sigma) / 2} v\right|_{2}^{2} .
\end{gathered}
$$

Substituting (101)-(102) into (100), we get that, for all $t \geq 0$,

$$
\begin{gathered}
\frac{d}{d t}\left[\left|A^{(1+\sigma) / 2} v\right|_{2}^{2}+\left(1-\mu \delta+\delta^{2}\right)\left|A^{(1+\sigma) / 2} u\right|_{2}^{2}\right] \\
+\omega\left[\left|A^{(1+\sigma) / 2} v\right|_{2}^{2}+\left(1-\mu \delta+\delta^{2}\right)\left|A^{(1+\sigma) / 2} u\right|_{2}^{2}\right] \\
\leq C\left\|v_{1}(t)\right\|_{0}^{2}\left[\left|A^{(1+\sigma) / 2} u\right|_{2}^{2}+\left(1-\mu \delta+\delta^{2}\right)\left|A^{(1+\sigma) / 2} v\right|_{2}^{2}\right] \\
+C\left(1+\left\|u_{t}(t)\right\|_{0}^{2(r-2)}\left\|u_{t t}(t)\right\|_{0}^{2}+C|g(x)|_{2}^{2}\right),
\end{gathered}
$$

where $\omega=\min ((\mu-\delta) / 2,2 \delta)$. Using the Gronwall inequality and integrating over $[0, t]$, we get that there exists a constant $M_{\sigma}(>0)$ which depends only on the $E_{\sigma}$-bounds of $B_{\sigma}$ such that, for any $t \geq 0$ and $z_{0} \in B_{\sigma}$,

$$
\|z(t)\|_{\sigma} \leq M_{\sigma} .
$$

In the following, based on Lemmas 11 and 12, we perform a bootstrap argument, whose proof is similar to that of Lemmas 11 and 12 (e.g., see $[11,13])$. Here we only point out the results and omit the proof.

Lemma 13. For each $\sigma \leq \theta \leq 1$, assume that $B_{\theta}$ is bounded in $H_{\theta}$. Then there exists a constant $M_{\theta}$ which depends only on the $H_{\theta}$-bounds of $B_{\theta}$, such that

$$
\left\|S(t) z_{0}\right\|_{\theta} \leq M_{\theta}
$$

holds for all $t \geq 0$ and $z_{0} \in B_{\theta}$. 
Lemma 14. For each $\sigma \leq \theta \leq 1-\sigma$, if the initial data set $B_{\theta}$ is bounded in $H_{\theta}$, then the decomposed ingredient $z_{c}=$ $\left(w(t), w_{t}(t)\right.$ (the solutions of (47)) satisfies

$$
\left\|z_{c}(t)\right\|_{\theta+\sigma}^{2} \leq \mathscr{J}_{\theta}
$$

which holds for all $t \geq 0$ and $z_{0} \in B_{\theta}$, where the constant $J_{\theta}$ depends only on the $H_{\theta}$-bounds of $B_{\theta}$.

Theorem 15 (asymptotic regularity of solutions). Let $\Omega$ be a bounded domain in $\mathbb{R}^{3}$ with smooth boundary and let $f$ satisfy (11)-(15). Assume further that $g(x) \in L^{2}(\Omega)$. Then there exist a bounded (in $E_{1}$ ) set $B_{1} \subset E_{1}$, a positive constant $v$, and a monotonically increasing function $Q(\cdot)$ such that, for any bounded (in $E_{0}$ ) set $B \subset E_{0}$ and $t \geq 0$, the following estimate holds:

$$
\operatorname{dist}_{E_{0}}\left(S(t) B, B_{1}\right) \leq Q\left(\|B\|_{0}\right) e^{-v t},
$$

where dist $E_{E_{0}}$ denotes the usual Hausdorff semidistance in $E_{0}$.

Proof. Let $B_{0}$ be a bounded (in $E_{0}$ ) absorbing set obtained in Theorem 5.

From Lemmas 11 and 7, we know that the set $A_{\sigma}=\{z \in$ $\left.E_{\sigma}:\|z\|_{\sigma} \leq \mathscr{J}_{0}\right\}$ satisfies

$$
\operatorname{dist}_{E_{0}}\left(S(t) B_{0}, A_{\sigma}\right) \leq Q_{1}\left(\left\|B_{0}\right\|_{E_{0}}\right) e^{-k_{0} t},
$$

where the constant $\mathscr{J}_{0}$ is given in Lemma 11 corresponding to $B_{0}$. Applying Lemmas 14 and 7 to $A_{\sigma}$, we see that there is a set $A_{2 \sigma}$ which is bounded in $E_{2 \sigma}$, such that

$$
\operatorname{dist}_{E_{0}}\left(S(t) A_{\sigma}, A_{2 \sigma}\right) \leq Q_{1}\left(\left\|A_{\sigma}\right\|_{E_{0}}\right) e^{-k_{0}^{\prime} t}
$$

where $k_{0}^{\prime}$ depends on the $E_{0}$-bounds of $A_{\sigma}$ (determined in Lemma 7).

Combining with Lemma 4, we know that the conditions in Lemma 1 are all satisfied. Hence, we have

$$
\operatorname{dist}_{E_{0}}\left(S(t) B_{0}, A_{2 \sigma}\right) \leq Q_{1}\left(\left\|B_{0}\right\|_{E_{0}}\right) e^{-k_{0}^{\prime \prime} t}
$$

for two appropriate constants $C$ and $k_{0}^{\prime \prime}$. Since $\sigma=$ $\min \{1 / 4,(5-p) / 2,(6-r) / 2\}$ is fixed, by finite steps (e.g., at most by $[1 / \sigma]+1$ steps), we can infer that there is a bounded (not only in $E_{0}$, but also bounded in $E_{1}$ ) set $B_{1} \subset E_{1}$ such that

$$
\operatorname{dist}_{E_{0}}\left(S(t) B_{0}, B_{1}\right) \leq Q\left(\left\|B_{0}\right\|_{E_{0}}\right) e^{-v t}
$$

Note further that all the constants in (111) depend only on $\left\|B_{0}\right\|_{0}$ and $|g|_{2}$. Now, for any bounded (in $E_{0}$ ) $B$, from Theorem 5 we see that there is a $T_{B}$ such that

$$
S(t) B \subset B_{0}, \quad \forall t \geq T_{B} .
$$

Hence,

$$
\operatorname{dist}_{E_{0}}\left(S(t) B, B_{0}\right) \leq M e^{-\gamma\left(t-T_{B}\right)}
$$

where $M=\sup \left\{\|S(t) B\|_{0}: 0 \leq t \leq T_{B}\right\}<\infty$. Finally, we apply the attraction transitivity lemma, that is, Lemma 1 , again to (111) and (113), and this completes the proof.

\section{Global Attractor}

Collecting now Theorem 5, Lemmas 7 and 8, and Theorem 15, we establish that $\{S(t)\}_{t \geq 0}$ is asymptotically compact. Therefore, by means of well-known results of the theory of dynamical systems we get the following.

Theorem 16. Let $\Omega \subset \mathbb{R}^{3}$ be a bounded domain with smooth boundary and assume that $f$ satisfies (11)-(15); the semigroup $\{S(t)\}_{t \geq 0}$ possesses a global attractor $\mathscr{A}$ on $H_{0}^{1}(\Omega) \times H_{0}^{1}(\Omega)$. Moreover, this attractor $\mathscr{A}$ is bounded in $H^{2}(\Omega) \times H^{2}(\Omega)$.

\section{Acknowledgment}

This work was partly supported by National Science and Technology Major Projects of China (no. 2012ZX10001001006) and the Scientific Research Fund of Hunan Provincial Education Department (Grant no. 10C042, 11A008).

\section{References}

[1] I. L. Bogolubsky, "Some examples of inelastic soliton interaction," Computer Physics Communications, vol. 13, no. 3, pp. 149155, 1977.

[2] P. A. Clarkson, R. J. LeVeque, and R. Saxton, "Solitary-wave interactions in elastic rods," Studies in Applied Mathematics, vol. 75, no. 2, pp. 95-121, 1986.

[3] C. E. Seyler and D. L. Fanstermacher, "A symmetric regularized long wave equation," Physics of Fluids, vol. 27, no. 1, pp. 58-66, 1984.

[4] W. G. Zhu, "Nonlinear waves in elastic rods," Acta Solid Mechanica Sinica, vol. 1, no. 2, pp. 247-253, 1980.

[5] J. Ferreira and N. A. Larkin, "Global solvability of a mixed problem for a nonlinear hyperbolic-parabolic equation in noncylindrical domains," Portugaliae Mathematica, vol. 53, no. 4, pp. 381-395, 1996.

[6] H. W. Zhang and Q. Y. Hu, "Existence and stability of the global weak solution of a nonlinear evolution equation," Acta Mathematica Scientia A, vol. 24, no. 3, pp. 329-336, 2004.

[7] H. A. Levine and J. Serrin, "Global nonexistence theorems for quasilinear evolution equations with dissipation," Archive for Rational Mechanics and Analysis, vol. 137, no. 4, pp. 341-361, 1997.

[8] H. A. Levine, P. Pucci, and J. Serrin, "Some remarks on global nonexistence for nonautonomous abstract evolution equations," in Harmonic Analysis and Nonlinear Differential Equations, vol. 208 of Contemporary Mathematics, pp. 253-263, American Mathematical Society, Providence, RI, USA, 1997.

[9] Y. Xie and C. Zhong, "The existence of global attractors for a class nonlinear evolution equation," Journal of Mathematical Analysis and Applications, vol. 336, no. 1, pp. 54-69, 2007.

[10] A. N. Carvalho and J. W. Cholewa, "Local well posedness, asymptotic behavior and asymptotic bootstrapping for a class of semilinear evolution equations of the second order in time," Transactions of the American Mathematical Society, vol. 361, no. 5, pp. 2567-2586, 2009. 
[11] V. Pata and M. Squassina, "On the strongly damped wave equation," Communications in Mathematical Physics, vol. 253, no. 3, pp. 511-533, 2005.

[12] V. Pata and S. Zelik, "Smooth attractors for strongly damped wave equations," Nonlinearity, vol. 19, no. 7, pp. 1495-1506, 2006.

[13] C. Sun and M. Yang, "Dynamics of the nonclassical diffusion equations," Asymptotic Analysis, vol. 59, no. 1-2, pp. 51-81, 2008.

[14] M. Yang and C. Sun, "Dynamics of strongly damped wave equations in locally uniform spaces: attractors and asymptotic regularity," Transactions of the American Mathematical Society, vol. 361, no. 2, pp. 1069-1101, 2009.

[15] S. Zelik, "Asymptotic regularity of solutions of a nonautonomous damped wave equation with a critical growth exponent," Communications on Pure and Applied Analysis, vol. 3, no. 4, pp. 921-934, 2004.

[16] P. Fabrie, C. Galusinski, A. Miranville, and S. Zelik, "Uniform exponential attractors for a singularly perturbed damped wave equation," Discrete and Continuous Dynamical Systems A, vol. 10, no. 1-2, pp. 211-238, 2004.

[17] L. C. Evans, "Partial differential equation," GSM 19, American Mathematical Society, Providence, RI, USA, 1998. 


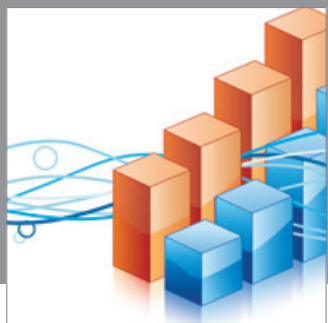

Advances in

Operations Research

mansans

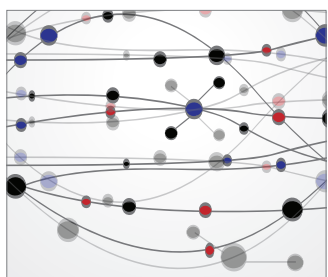

The Scientific World Journal
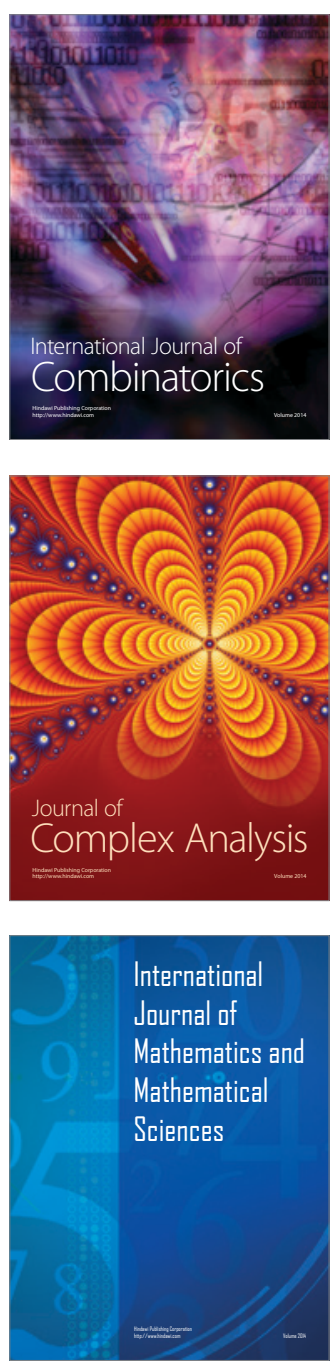
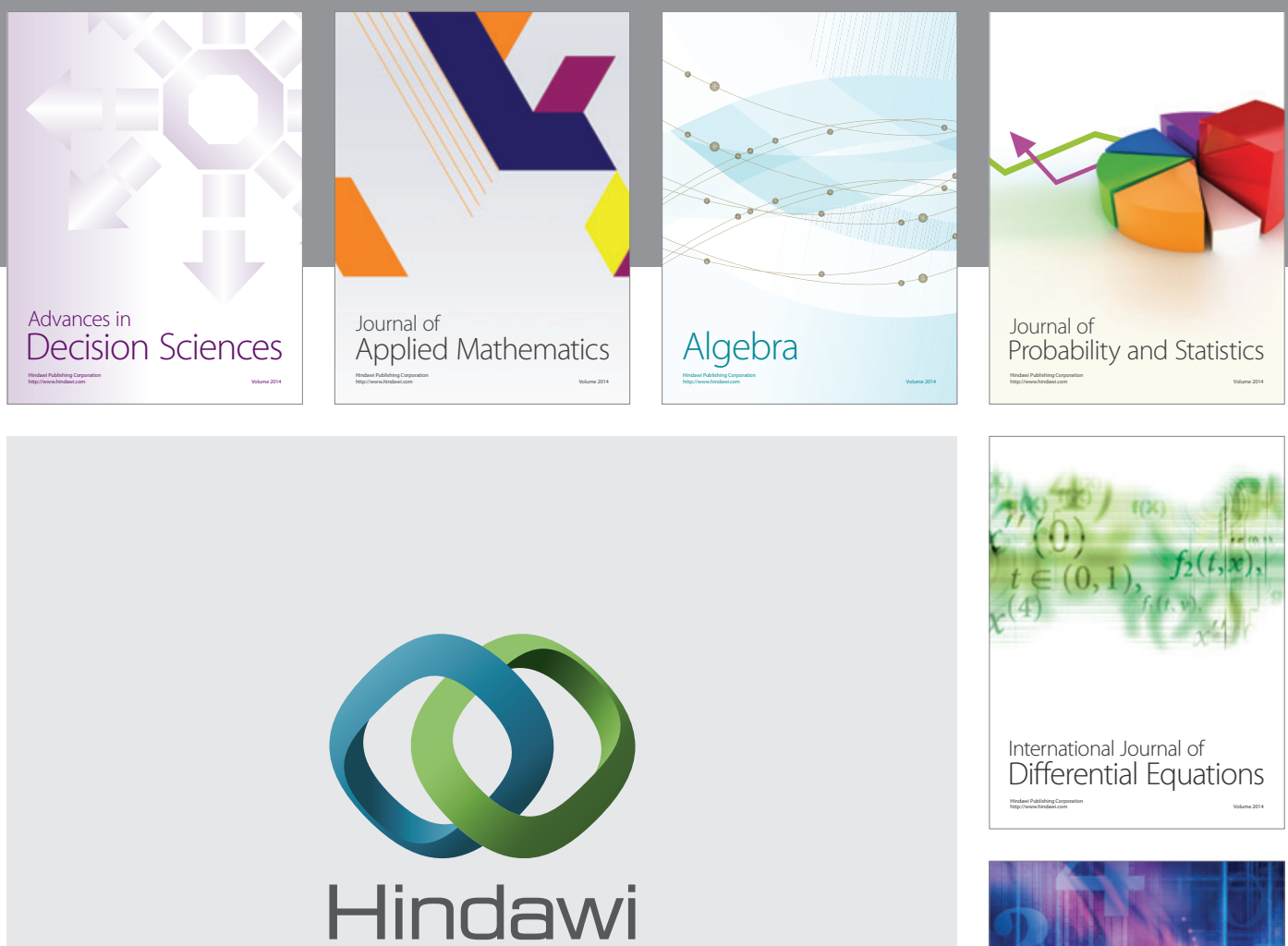

Submit your manuscripts at http://www.hindawi.com
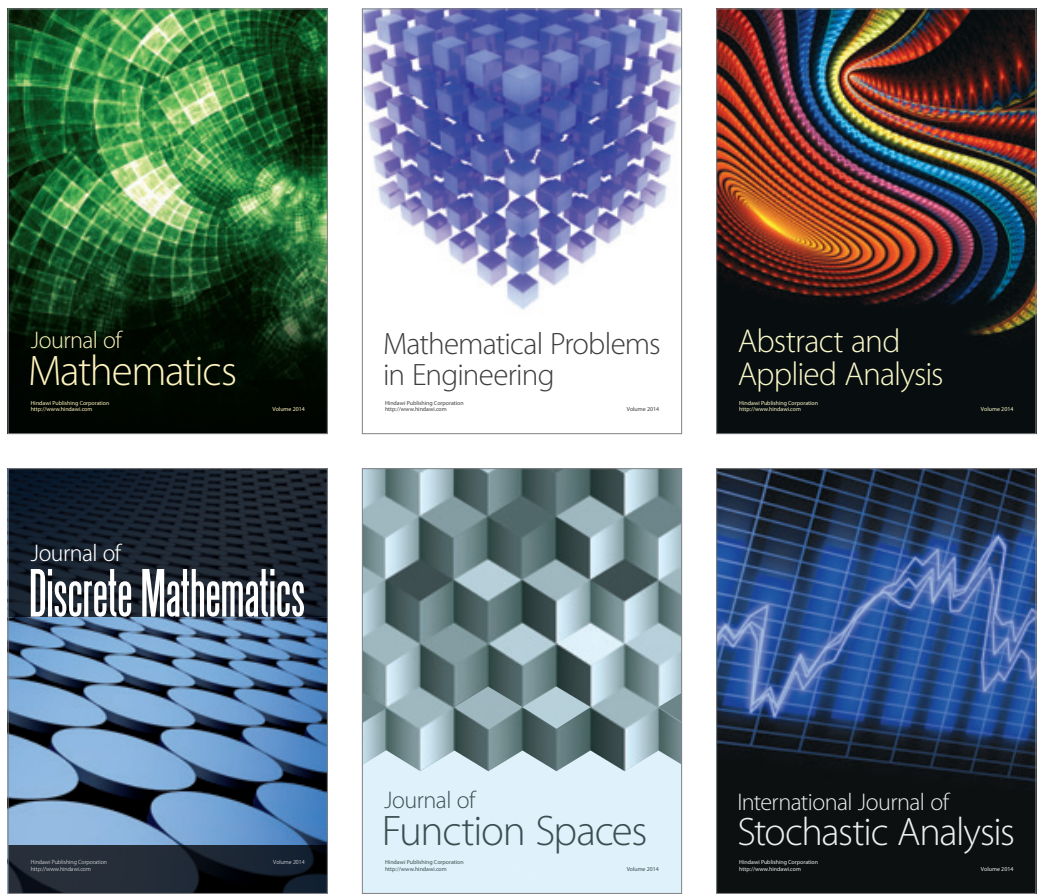

Journal of

Function Spaces

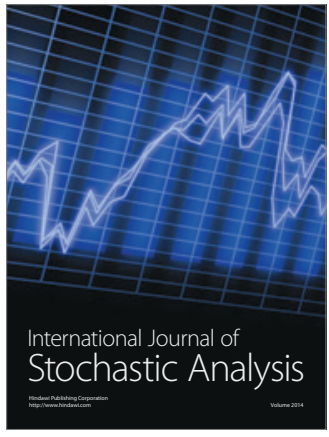

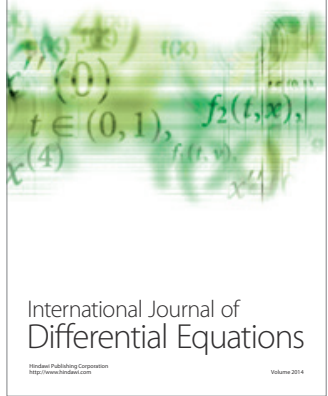
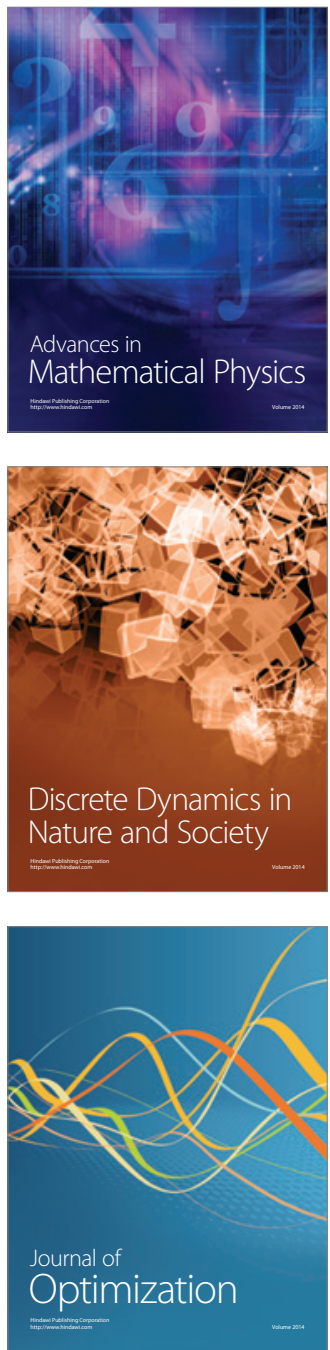